\title{
Entrevista com Antonio Arellano Hernández ${ }^{\star}$
}

\author{
Entrevistadores: Clovis Dorigon ${ }^{\star \star}$ Ivan da Costa Marques ${ }^{\star \star \star}$ \\ e Marcia Moraes ${ }^{\star \star \star \star}$
}

Entrevistadores: No texto "La genética de Edwin Wellhausen y la irreproducibilidad en México de la hibridación norteamericana del maiz" (ARELLANO, 2005), o senhor argumenta que para a introdução das técnicas de hibridação do milho no México, o melhorista americano Wellhausen, teve que "criar" grupos sociais até então inexistentes neste país, como o de agricultores capitalistas especializados na produção de sementes híbridas, selecionados dentre os mais tecnificados. Isto poderia nos levar a concluir que, assim como o social define o técnico, inverso também é verdadeiro, ou seja, que neste caso, o técnico moldou o social?

Arellano: Lo que he querido demostrar en este libro es, primeramente, que en los espacios de la investigación lo técnico es simultáneamente social y viceversa.

Ahora bien, si se considera que la transferencia tecnológica es un proceso de construcción de objetos y sujetos y no un simple traslado de objetos concluidos hacia nuevos contextos, entonces el estudio de la transferencia tecnológica permite mostrar este proceso sincrónico.

En seguida, he querido mostrar que la tecnicidad se despliega en esquemas de producción sociotécnica (EPST) ${ }^{1}$ (GILLE, 1978, p. 112), mismos que de inspiración en Foucault, hoy les reformulo como dispositivos. La idea de EPST es importante pues muchos investigadores influenciados por la Teoría del Actor-red se han concentrado en estudiar la construcción de artefactos y conocimientos en sí mismos pero han prestado poca atención a la producción del esquema que permite su elaboración. En el libro no se trata de explicar como se puso en escena un artefacto, como si se estudiara como Daniel Bell inventó el teléfono, o como surgió la vacuna contra el ántrax, o de cualquier artefacto o conocimiento sino de cómo se logró que estas invenciones formaron esquemas de producción sociotécnica; en este sentido el EPST está más cerca de la noción de habitus, en el sentido de acto

* Profesor e Investigador del Laboratório Tecnociencia-sociedad de la Facultad de Ciencias Políticas y Administración Pública. Universidad Autónoma del Estado de México. Ciudad Universitaria, Toluca, México. CP 50100.

E-mail:aah@uaemex.mx

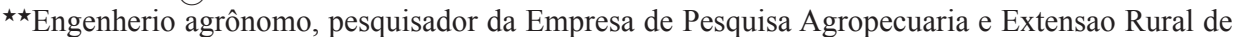
Santa Catarina (Epagri), mestre em Desenvolvimento, Sociedade e Agricultura pela UFRRJ/CPDA e doutorando em Engenharia de Produção pela UFRJ/Coppe.

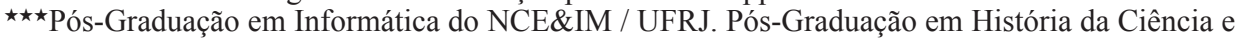
da Técnica e Epistemologia / UFRJ

Email: imarques@nce.ufrj.br

$\star \star \star \star$ Professora do Programa de Pós-graduação em Psicologia da UFF; Editora da Revista do Departamento de Psicologia-UFF.

Email:mmoraes@vm.uff.br 
operatorio de Marcel Mauss, de sistema técnico como diría Bertrand Gilles o de la noción de sistema tecnológico del monumental trabajo de Thomas Hughes sobre la construcción de las redes de electrificación en Estados Unidos.

He mostrado que el esquema de producción sociotécnico de producción de semillas híbridas propuesto por Wellhausen requería de dos acciones técnicas complementarias; primero conducir la purificación racial de ciertas líneas de maíz (milho) seleccionadas por los intereses productivos de los investigadores y agricultores y luego cruzar estas líneas para formar semillas y plantas híbridas. Pero estas acciones técnicas eran simétricas de las acciones sociales necesarias para llevar a término el proyecto.

En efecto, antes del mejoramiento genético wellhauseniano, los agricultores de maíz eran simultáneamente productores de grano y semilla, entonces hay que notar que aquí la noción agricultores de maíz es una noción sociotécnica. Pero el aspecto social de la hibridación propuesto por Wellhausen requería simétricamente la purificación y la cruza de los agricultores. El desafío de Wellhausen consistía en fabricar las semillas híbridas y en el mismo proceso crear a los colectivos que las produjeran, sembraran y consumieran.

Por un lado, debía ser capaz de convencer a ciertos agricultores para que se especializaran en producir semillas, para lo cual debían consagrar sus tierras y sus recursos a la producción comercial de semillas híbridas y asegurarles su semilla adquiriría un precio comercial y que otros agricultores iban a comprar sus semillas; además como muestra de esa confianza, él les proporcionaría las muestras y toda la asesoría técnica con las que se podría escalar la producción de dichas semillas. Pero también debería ser capaz de convencer a otros agricultores a especializarse en la producción de grano, a hacerles ver, desde ahora, que la semilla era un insumo comercial y que por lo tanto adquiría un precio, que debería confiar en que esas semillas las produciría otro agricultor, que sembrándolas incrementaría su producción al grado de obtener una ganancia extraordinaria y suficiente para pagar el precio de las nuevas semillas mejoradas.

Dicho en términos marxistas, se trataba de convertir en el mismo hecho un bien de uso en mercancía y una relación socio-natural en una comercial y, de replicar en México lo realizado en Estados Unidos, algunas décadas atrás.

Luego entonces, cuando Wellhausen intentó replicar el Land-Grant-System ${ }^{2}$ para el maíz tuvo que crear un esquema de producción técnica y los colectivos sociales correspondientes, pero la historia nunca se repite y lo sucedido fue la construcción de un esquema de producción de semillas y de agricultores único e irrepetible. La prueba de esto es que aún hoy existen agricultores productores de grano-semilla, productores de semillas mejoradas y productores de híbridos como no existen en Estados Unidos.

Entrevistadores: Em seu texto sobre a hibridação do milho, o senhor mostra os problemas socioeconômicos e ambientais causados pela introdução de uma tecnologia exógena, desenvolvida para as condições norte-americanas em um país periférico, no caso o México. Quais seriam as principais contribuições da Teoria 
do Ator-Rede para se pensar políticas tanto multilaterais como nacionais visando o desenvolvimento de políticas de ciência e tecnologia que possibilite diminuir estas assimetrias entre países desenvolvidos e periféricos?

Arellano: El principio de simetría de David Bloor aplicado en el estudio de las controversias de nuestros países, a propósito de transferencias tecnológicas, se traduciría en no ser nacionalista ni imperialista, ni centralista ni periferista, no darles la victoria a los desarrollados en detrimento de los subdesarrollados. En este sentido lo que he querido mostrar en el libro del que hablamos es que las asimetrías científicas y tecnológicas entre países desarrollados y periféricos (manteniendo los términos que valdría la pena criticar en algún momento) son inevitables y que no necesariamente es mejor seguir los caminos marcados por los actores llamados desarrollados; que es pertinente estar al tanto de sus avances pero saber desarrollar líneas originales de investigación propias.

En este libro mostramos una controversia científica, tecnológica y política entre dos líneas de desarrollo tecnológico que se ha extendido durante más de 60 años, por un lado Wellhausen y sus híbridos de maíz y de otro Taboada y sus variedades estables genéticamente (similares a las de los agricultores tradicionales pero racionalizada su selección). Los promotores de los híbridos han sostenido que no hay marcha atrás en la evolución social mas que la realización capitalista de la agricultura y convertir a las semillas en un bien de cambio, sobre todo con agricultores que disponen de buenas tierras y máquinas agrícolas; en tanto que los promotores de las variedades estabilizadas han apelado a la imposibilidad de convertir a las semillas en negocio cuando las condiciones ecológico-productivas de los agricultores son inestables y cuando los mismos agricultores son un mosaico de culturas y los usos del maíz son múltiples. Con esto se comprueba que la diversidad biológica del maíz es solidaria de la diversidad cultural.

Al final de cuentas y luego de muchas investigaciones sobre adaptación de vegetales, otros agrónomos se han transformado en sociotecnólgos regionalistas mostrado que los híbridos y las variedades estabilizadas tienen un área técnicoproductiva y social específica y, aún más, estos agrónomos y muchos ecologistas reclaman la no intervención de la ciencia y tecnologías modernas concretadas en semillas para salvaguardar la riqueza genética del maíz (las llamadas zonas de conservación in situ).

Pero hay más, los híbridos de maíz desarrollados por Wellhausen no tenían casi nada que ver con los híbridos americanos. Estos se parecían más a las variedades estabilizadas del equipo mexicano de Taboada. En otro texto, he aludido a las enormes dificultades de reproducción de los esquemas de producción sociotécnica, es decir que los intentos de reproducir y transferir los EPS-T representan verdaderos desafíos para los actores interesados pues los actores que funcionan como ambiente no corresponden a los mecanismos de acción de las tecnologías donde fueron desarrolladas originalmente (Cf. ARELLANO, 2005).

Si consideramos que la tecnicidad es un complejo de temas técnicos, sociales, ambientales e intersubjetivos, este libro puede servir para criticar la asimetría existente en los encargados de las políticas públicas de ciencia y tecnología de los 
países latinoamericanos por los intentos de solucionar con importaciones de artefactos problemas de orden económico y social. Sí consideramos que los artefactos son simétricos a los colectivos sociales, al ambiente natural y a la situación intersubjetiva, entonces los políticos de la ciencia y la tecnología de nuestros países deberían asumir la creación de situaciones en las que los problemas se confronten mediante soluciones conformadas por arreglos heterogéneos compuestos por colectivos, artefactos, símbolos e intesubjetividades.

Si los políticos responsables de las líneas de acción científicas de los países llamados periféricos aceptan e imponen la linealidad del desarrollo de conocimientos y artefactos estarán condenando a sus países al eterno subdesarrollo por dos razones principales: en primer lugar, estarán aceptando la imposibilidad de alcanzar a los países desarrollados en la línea que ellos van siempre adelante y por lo tanto siempre se vivirá la situación de subdesarrollo y, en segundo lugar, estarán admitiendo una visión asimétrica entre los artefactos y los colectivos sociales y sus perspectivas se acotarán inmediatamente al determinismo según el cual los artefactos determinan lo social. Ambas razones nos conducirían permanentemente a copiar tardíamente lo realizado en el "centro" y nuestra ciencia y tecnología serían acciones de adaptación de principios generales a las situaciones concretas de los países y regiones.

Empleando críticamente la teoría del actor red, se pueden repensar las políticas de ciencia y tecnología tomando en consideración que las asimetrías entre los países son resultado de las simetrías objetos-sujetos y por lo tanto se construye la necesidad de edificar nuestras propias simetrías entre colectivos-artefactosintersubjetividades-ambientes.

Aplicando críticamente la noción de actor-red estamos en posibilidades de imaginar la invención de países diferentes, países red, países que deberían enfrentar el mundo actual como les es dado asumiendo la enorme dificultad de crear redes alternas, propias y viables y que el reto es socio-técnico-natural-intersubjetivo.

No aplicar críticamente la noción de actor-red sería una tautología pues desde las ciencias sociales estaríamos replicando tardíamente una noción que tiene su propio plexo socio-técnico.

Entrevistadores: Na visão de autores como Michel Callon, Bruno Latour e John Law os "atores-redes" que habitam uma versão da "realidade" são "simultaneamente reais como a natureza, narradas como o discurso e coletivos como a sociedade."

Arellano: En general, estoy de acuerdo con la afirmación, pero los investigadores inspirados de estos autores adoptan en pocas ocasiones un compromiso con la simultaneidad del tejido reticular de naturaleza-significantes-colectivos.

Es bien interesante que una parte de detractores de la teoría del actor-red se mofan de la afirmación según la cual los artefactos y los textos son actores. Estas burlas evidencian dos incomprensiones: por un lado suponen que los humanos actúan bajo su libre albedrío (digamos, en el mundo de la libertad) y sin las restricciones y posibilidades que les brindan los artefactos y los símbolos (fuera 
del mundo de la necesidad). Por otro, consideran, paradójicamente, que los artefactos y las construcciones simbólicas tienen posibilidades de acción pero les llaman posibilidades causales. Esto último es muy simpático pues algunos críticos de la TAR que se ríen de la posibilidad de que los artefactos sean actores, asumen que los artefactos y símbolos impactan a la sociedad... prueba de ello son los innumerables ejemplos de estudios que estudian los impactos de la tecnología o de la cultura simbólica en la sociedad.

El malentendido consiste en que no es fácil aceptar que para la TAR, ninguna entidad es autónoma; así, ni los humanos, ni los artefactos, ni los textos, ni las intersubjetividades (agregaría yo) actúan por sí solos. Con la TAR nos colocamos en una situación más realista en la que los humanos actúan restringidos y posibilitaos por los artefactos, por los actos lingüísticos de otros actores y por las construcciones intersubjetivas. Por su parte, los artefactos actúan exclusivamente en interrelación con los humanos (por ejemplo, un automóvil abandonado en la selva regresa a su estado salvaje, sufre las consecuencias del medio natural y deja de ser propia y simbólicamente un automóvil). Los símbolos son mediadores y constituyentes de la intersubjetividad cuando actúan como representaciones de la acción de los humanos sobre los artefactos y de la construcción intersubjetiva de los actores humanos. Finalmente, la intersubjetividad permite la construcción mediada por símbolos y artefactos de los humanos, las intersubjetividades movilizan a los humanos de acuerdo con las posibilidades inscritas en los artefactos y textos.

Pero vale la pena aclarar que no creo que los autores fundadores de la TAR se propongan erigirse como los representantes de un método auténticamente realista, ese es un problema epistemológico mayor que escapa a su ámbito. En el texto de John Law (2004) After method hay una apología por los métodos combinados y en el Texto de Latour (1999) sobre la crisis de objetividad o crisis ecológica no se llega a plantear la idea de que la TAR pudiese ser un método realista, aunque si se reconoce que la noción de red es un recurso epistemológico para criticar lo poco real que resulta la acción de los humanos al margen de los artefactos, textos e intersubjetividades... agregaría yo.

Ahora bien, la frase "reais como a natureza, narradas como o discurso e coletivos como a sociedade" me parece limitada, a mi juicio abria que agregar que son compartidos como la intersubjetividad, incorporar esta dimensión es algo muy complicado y requerirá de trabajos sobre este campo.

Entrevistadores: Você afirma que "por difícil que pareça, a noção de rede foi deglutida pelas tendências dominantes do estruturalismo" e propõe que se revisite a noção de "dispositivos" de Michel Foucault que, "en sentido metodológico, compreenderiam elementos de caráter institucional, simbólico e material", elementos aos quais você agrega a "simultaneidade."

Arellano: Bueno, la simultaneidad se refiere a una especie de antídoto contra el determinismo pues si las cosas ocurrieran asincrónicamente habría que considerar que un elemento ocurre primero y sirve de soporte o de causa o condición para los otros. Sí las instituciones soportan la construcción simbólica del mundo como aducen algunos abogados esto es cierto pero incompleto y por poco realista. 
Si consideramos que el lenguaje es el que sirve para inscribir las instituciones como aducirían algunos lingüistas estaríamos reconociendo una realidad pero igualmente incompleta, y si consideramos que las maquinas de escribir o computadoras soportan las instituciones y la construcción simbólica estaríamos en el mismo tenor de referirnos a una realidad incompleta. Por esta razón la simultaneidad es importante pues los juegos ocurren siempre en diferentes dimensiones aunque nuestra capacidad disciplinaria nos permita vislumbrar e intelegir sólo algunos aspectos actuando sobre otros. El problema, en todo caso es nuestra formación disciplinaria que nos impide reconocer el tipo de acciones mezcladas que realizamos cotidianamente. Seguramente a oídos de ciertos premodernos, nuestras estrategias epistemológicas solo sean pérdida de tiempo, en tanto que para ellos las cosas ocurren en una sola dimensión compleja de una realidad que no admite las divisiones que nos entretienen debido a nuestras formaciones disciplinarias.

Pero hay una cuestión importante. Si circunscribimos a estas dimensiones (material, simbólica y social) el mundo real sigue aún muy fracturado por esta razón he planteado en el texto que se publica en este número que es importante ir más allá de plantear las tres famosas dimensiones. He planteado en el texto ¿Es posible una epistemología política que solucione la asimetría entre naturaleza absolutizada y politica relativizada? (ARELLANO, no prelo) La necesidad de considerar las dimensiones mínimas que han estado presentes en el proceso de hominización y en este sentido me parece que habría que considerar la necesidad de incluir la dimensión de la intersubjetividad como proceso simultáneo a la construcción colectiva, simbólica y social. En todo caso, con estos cuatro elementos estaríamos más cerca de lo que llamo una matriz antropológica como sustento epistemológico.

Mi suposición es que con la noción de dispositivo podemos acercamos a estos cuatro elementos con una mayor libertad de investigación, en lugar de ceñirnos a las tres dimensiones señaladas en la TAR.

Entrevistadores: Como se assemelham e se diferenciam cada uma das características abaixo nos atores-redes e nos dispositivos de Foucault:

1) reais como a natureza $<$ inscrição $<>$ material;

2) narradas como o discurso $<>$ simbólico $<>$ material-organizacional;

3) coletivo como a sociedade $<>$ efeito social $<>$ institucional?

Arellano: Lo que yo he hecho es realizar una lectura de Foucault que tenía pendiente de hacer pues había asumido, como muchos, la metáfora del poderconocimiento. Hoy me parece que la noción de dispositivos en Foucault es un término pleno de contenido científico-técnico y sobre todo epistemológico.

Foucault no ha sido leído por los autores del movimiento ciencia-tecnologíasociedad y las lecturas de los fundadores del movimiento TAR ha sido hechas para discutir cuestiones conceptuales, así Law (2000) se refieren a él para comparar ciertas lecturas de la biopolítica en relación a nuevas prácticas sobre el cuerpo, Callon (1991) se ha referido a dispositivo como una noción instrumental de $\operatorname{red}^{3} \mathrm{y}$ 
Latour ha criticado someramente la ausencia de relación entre símbolos y realidad (las palabras y las cosas) y el hecho de que a diferencia de Fleck, la construcción de un hecho científico no haya sido tomado en cuenta por Foucault (Cf. LATOUR; HERMANT, 1998; LATOUR, 2005a).

El punto es que sí Foucault había escrito sobre tecnología como tecnología del castigo sobre el cuerpo, bien valía la pena leerlo para aprender sobre sus nociones de tecnología y de la voluntad de saber. Y lo que encontré de esta lectura diagnóstica (siguiendo la recomendación de Althusser) fue que las nociones triádicas de la noción de red de la TAR (naturaleza, discurso y sociedad) podrían, con mucha precaución y reservas compararse heurísticamente con las nociones triádicas, también, de la noción de dispositivo de lo que resultaba una traducción a los elementos foucaultianos en las dimensiones de moral, teoría y derecho. Esta heurística es visible incluso en la forma de relativizar las entidades triádicas cuando Foucault escribe: a los dispositivos "no se los puede reducir ni a teorías del derecho (aunque coinciden con ellas) ni identificarlos a aparatos o instituciones (aunque se apoyen en ellos) ni hacerlos derivar de opciones morales (aunque se encuentre en ellas su justificación)" (FOUCAULT, 1976, p. 155) y Latour emplea las comparaciones de manera positiva empleando el conector "como", cuando escribe "reais como a naturaza, narradas como o discurso y coletivo como a sociedade". Pero además de esta heurística, el contenido es por demás poderoso en Foucault cuando escribe: las tecnologías del poder de castigar pueden leerse como los cambios en las propiedades instrumental-materiales, simbólicas y sociales, encontrando que en cada uno de esos tres dispositivos nos encontramos con elementos instrumentalesmateriales del castigo (ceremonias, sujetos de derecho y cárceles), elementos de inscripción de las representaciones (marcas, signos y trazas) y efectos sociales (aterrorizamiento, manipulación de representaciones y sometimiento).

En la lectura no se trataba de buscar similitudes entre la noción de red y dispositivo sino de hacer heurística y buscar las posibilidades abiertas por Foucault y, luego de toda esta lectura encuentro que las ventajas de la noción de dispositivo consisten en que permitiría investigar situaciones de alta complejidad no previstas por la noción de red, tales como la investigación trasnacional que involucra niveles tecnológicos y países pero que de alguna manera están interactuando; la investigación multiobjetivos que va más allá de la transdisciplinariedad como los casos de la medicina en la que se ponen en contacto la investigación etnográfica del uso de remedios locales, con la investigación farmacéutica, la investigación clínica, etc.

Asimismo, la noción de dispositivo está mas cerca de la investigación de los esquemas de producción sociotécnica, así, el término biopolítica alude a un proceso de construcción de la política de la vida en la que el conocimiento juega un papel crucial, evidenciando que Foucault no escribió sobre la construcción de un hospital o de un sistema arquitectural sino del nacimiento de la clínica cercano a la noción de esquema de producción.

La otra gran ventaja de la noción de dispositivos es su interés analítico o epistemológico por sobre las ambiciones nomológicas, descriptivas. En la TAR se han tenido que hacer seminarios para explicar la necesidad de avanzar en la versión 
metodológica de la noción de red y Latour ha tenido que publicar su Introducción to Actor-Network Theory apenas en el 2005.

$\mathrm{Y}$, finalmente la noción de dispositivo surge del posestructuralismo foucaultiano, situación que es un gran mérito si consideramos que la noción de red está tan extendida que autores tan influyentes como Bourdieu y Castells escriben sobre redes pero las explican repletas de estructuras.

En fin, la noción de dispositivo puede ser una noción provisional para acercarse de manera más clara a la idea de matriz antropológica como sustento epistemológico, que es el centro de mi interés académico actual.

Entrevistadores: Que diferenças você apontaria entre os atores-redes e os dispositivos de Michel Foucault de modo colocar os últimos a salvo das "tendências dominantes do estruturalismo"?

Arellano: No creo que la noción de redes ni la de dispositivos se encuentren a salvo del estructuralismo o del posmodernismo o de alguna otra corriente epistemológica.

El asunto es muy importante pues el debate que se podría levantar en torno a la noción de red y de dispositivo sería una disputa entre investigadores que rechazan la epistemología arquitectural del estructuralismo como el relativismo epistémico del posmodernismo.

Esto es muy interesante pues pareciera que las preocupaciones epistemológicas de los grupos aglutinados en métodos reticulares (red o dispositivo) en Europa y Latinoamérica son diferentes y asimétricas; mientras allá las nociones reticulares se vean amenazadas por el posmodernismo como puede constatarse por la vehemente defensa de Latour hecha por Wallerstein (2001) en Conocer el mundo, saber el mundo. El fin de lo aprendido mostrando por que en Nunca hemos sido modernos (LATOUR, 1993) no hay rastros de posmodernismo. En América latina, el extenso uso del estructuralismo de Bourdieu y otros pueda ser visto como una amenaza a las nociones de red. En todo caso, mi preocupación es la extensa difusión que aún tiene el estructuralismo en nuestros países.

El asunto es que Latour esta más preocupado por el papel relativisador del posmodernismo que por el papel reductivo del estructuralismo. Esto puede apreciarse cuando a propósito del texto de Latour (2005b) intitulado emblemáticamente Reassembling the social. An introduction to actor-networktheory, Latour me ha comentado 4 que se trataba de retomar las posibilidades asociacionistas de la noción de red y que esta idea se encontraba en las nociones de Gabriel Tarde, que mucha gente estaba hablando de red pero que a pesar del sitio de John Law sobre los recursos de la TAR, no se había explicado la noción de red y era impostergable presentar una introducción.

En esa ocasión le comenté a Bruno que por que no avanzar por el camino iniciado por Foucault con la noción de dispositivo pero la respuesta fue que era muy ambigua su posición. En este sentido, considero que por las raíces postestructuralistas de dispositivo podría ser una noción mejor ubicada contra el 
estructuralismo hasta nuestros días demasiado extendido en nuestra región latinoamericana. El texto que hoy se publica no es una respuesta completa a Latour sobre su posición sobre la noción de red ni sobre la TAR pues las problemáticas de ellos no son las mismas que las de otros grupos, como el nuestro en México.

Entonces no se trata de proteger a la noción de red con la de dispositivo del estructuralismo sino de dar un aliento a la investigación que se propone un camino que privilegie lo epistemológico (o analítico como diría Foucault) y no una ambición conceptual (nomológica como diría Foucault).

Entrevistadores: Do seu ponto de vista, qual a importância dos enfoques de Callon e Latour para o campo das chamadas ciências humanas?

Arellano: La importacia de los enfoques de latour a las ciencias humanas es que las hace salir del humanismo antropocéntrico para plantear un humanismo acotado por su interrelación irrevocable con los artefactos y la naturaleza. La irrupción de los no-humanos en las ciencias humanas, es importante pues se podría pensar que el humanismo debería ser una radicalización de los aspectos en los que los artefactos, los conocimientos y la naturaleza se desprendían de lo humano.

En términos de este humanismo mediado por objetos y símbolos, el mundo actual es calloniano-latouriano pues mientras aparecen síntomas de la acción de la naturaleza, como el calentamiento global, nuevos colectivos denuncian la desnaturalización del mundo, convocan a la defensa de la naturaleza pero claman el involucramiento de nuevos colectivos (científicos naturalistas y humanistas) y mientras se acelera el diluvio de artefactos sobre la sociedad nuevos colectivos denuncian la deshumanización del hombre, convocan contra el consumismo mediante el consumo de todos los medios técnicos a su alcance. De estos dos movimientos, la idea es que las ciencias humanas deberían incorporar a los elementos que median la interacción de los humanos.

Es importante comentar que existe una paradoja en el trabajo latouriano pues mientras sus estudios sobre la tecnociencia se despliegan por múltiples ámbitos e intereses, su esfuerzo epistemológico se restringe a la saturación de la relación hombre naturaleza. Su epistemología es humanista cuando rechaza el exilio de los objetos de la relación con los humanos pero es inicial pues se requeriría de una epistemología descentrada en los humanos para operarla en otras dimensiones. Latour ha iniciado un trabajo que valdría la pena continuar, digamos que es una epistemología inicialmente interesante para proseguir su desarrollo y esto es tarea de muchos investigadores. 


\section{Notas}

${ }^{1}$ Bertrand Gille, postulaba en l'histoire des techniques (1978), que la técnica tomada en su conjunto constituye un sistema del cual las partes son interdependientes y considera que cada civilización corresponde a un sistema técnico.

${ }^{2}$ caso del transfert de tecnología como el que describí, es muy claro que el mejorador de semillas americano Wellhausen trataba por todos los medios de replicar un EPST que los americanos habían puesto en escena desde los años 30 del siglo XX y que fue conocido como Land-GrantSystem; este esquema consistía en una organización secuencial de enseñanza-investigacióntransferencia de tecnología agrícola. Wellhausen conocía este Sistema a la perfección pues había sido formado en él.

${ }^{3}$ Ver la noción de dispositivo empleado por Callon (1991, p. 195-230).

${ }^{4}$ Conversación realizada en enero de 2006.

\section{REFERÊNCIAS}

ARELLANO, H. A. ¿Es posible una epistemología política que solucione la asimetría entre naturaleza absolutizada y política relativizada? In: LÓPEZ, B. C. (Org.). Filosofía política contemporánea. México: UNAM. No prelo.

. La genética de Edwin Wellhausen y la irreproducibilidad en México de la hibridación norteamericana del maíz. In: ARELLANO, A. et al. (Comp.). Ciencias agrícolas y cultura cientifica en América Latina. Buenos Aires: Prometeo, 2005. p. 45-77.

CALLON, M. Réseaux technico-économiques et irréversibilités. In: BOYER, R.; CHAVANCE, B.; GODARD, O. (Org.). Les figures de l'irreversibilité en économie. Paris: EHESS, 1991. p 195-230.

FOUCAULT, Michel. Vigilar y castigar. México: Siglo XXI, 1976.

GILLE, B. L'histoire des techniques. Paris: Gallimard, 1978.

LATOUR, B. Nunca hemos sido modernos: ensayo de antropología simétrica. Madrid: Debate, 1993.

. Politiques de la nature: comment faire entrer les sciences en démocratie. Paris: La Découverte \& Syris, 1999.

. Postface a Ludkwik Fleck, génese et développemente d'un fait scientifique. Paris : Médicine et Sciences Humaines, 2005a.

. Reassembling the social: an introduction to actor-network-theory. Oxford: $\overline{\text { Oxford University Press, } 2005 \mathrm{~b} .}$

; HERMANT, É. Paris Ville Invisible: les empêcheurs de penser en ronde. Paris: La Decouverte, 1998.

LAW, J. After method: mess in social science research. London: Routledge, 2004.

. On the subject of the object: narrative, technology, and interpellation. Configurations, Baltimore, v. 8, n. 1, p. 1-29, winter 2000.

WALLERSTEIN, I. Conocer el mundo, saber el mundo: el fin de lo aprendido: una ciencia social para el siglo XXI. México: Siglo XXI, 2001. 